\title{
Mitral regurgitation in Dachshund dogs without heart murmurs
}

\author{
Magdalena Garncarz ${ }^{1}$, Marta Parzeniecka-Jaworska ${ }^{1}$, Magdalena Hulanicka ${ }^{2}$, \\ Michał Jank $^{3}$, Olga Szaluś-Jordanow ${ }^{4}$, Anna Kurek ${ }^{5}$ \\ ${ }^{1}$ Department of Pathology and Veterinary Diagnostics, ${ }^{2}$ Department of Physiological Sciences, \\ ${ }^{4}$ Department of Small Animal Disease with Clinic, \\ Faculty of Veterinary Medicine, Warsaw University of Life Sciences, Warsaw, 02-766 Warsaw, Poland \\ ${ }^{3}$ Veterinary Institute, Faculty of Veterinary Medicine and Animal Sciences, \\ Poznań University of Life Sciences, 60-637 Poznań, Poland \\ ${ }^{5}$ Department of Animal Breeding, Faculty of Animal Science, \\ Warsaw University of Life Sciences, 02-786 Warsaw, Poland \\ magdalena_garncarz@sggw.pl
}

Received: May 26, 2017

Accepted: August 24, 2017

\begin{abstract}
Introduction: Older small breed dogs are considered at risk for heart failure secondary to chronic mitral valve disease. However, few data are available on the onset of this disease in such dogs. This study was performed to determine if auscultation alone can be used to eliminate clinically relevant mitral valve regurgitation seen in echocardiography in Dachshund dogs. Material and Methods: Clinical and echocardiographic data were obtained from 107 dogs without heart murmurs. Results: The study revealed that $63.6 \%$ of the dogs had mitral regurgitation. Numbers increased with age and a larger percentage of male Dachshunds were affected than female Dachshunds. Mitral valve prolapse and thickening were mild, and the regurgitant area inextensive in most dogs. Conclusions: The study shows that mitral valve regurgitation is prevalent $(63.6 \%)$ in Dachshunds without heart murmurs. Typical lesions often become apparent during echocardiographic examinations in dogs under 5 years of age.
\end{abstract}

Keywords: dogs, Dachshund, heart murmur, echocardiography, mitral valve.

\section{Introduction}

Chronic mitral valve disease (CMVD) is characterised by myxomatous lesions of the mitral valve leaflets $(1,11)$ resulting in leaflet misalignment and regurgitation $(11,13)$, chordae tendineae lengthening and possibly chordal rupture $(8,19)$, left atrial enlargement, and left ventricular overload hypertrophy. These changes are easily recognisable with echocardiography. Clinical examination usually reveals a heart murmur in dogs with significant CMVD and a relationship between murmur intensity and disease severity has been documented $(1,3,4,11,13)$. Arrhythmias, especially supraventricular, may accompany the disease (17). Subclinical disease has been noted in the Dachshund $(7,16)$ and other breeds (23); however, the prevalence of heart disease in dogs without heart murmurs is unknown.
During previous studies related to genomic profiling (7) and plasma miRNA expression (10) in Dachshunds with CMVD it was difficult to find dogs without lesions and/or regurgitation in the echocardiographic examination in order to form a control group of healthy dogs. This prompted the authors to investigate the prevalence of these changes in clinically unaffected dogs, i.e. those without heart murmurs. This study is aimed at showing the prevalence of mitral regurgitation seen during echocardiography in Dachshunds without heart murmurs.

\section{Material and Methods}

A retrospective data analysis was carried out on Dachshund dogs presented for a cardiological 
examination at the Faculty of Veterinary Medicine, Warsaw University of Life Sciences $(n=404)$. Initially, 124 Dachshund dogs without heart murmurs were selected from the primary group of 404 symptom-free Dachshund dogs that had undergone full clinical and echocardiographic examinations. However, 17 dogs were eliminated from further analysis as a result of difficulty in auscultation because of obesity or muffled heart sounds for reasons other than obesity, leaving 107 dogs in the final study. This group consisted of 67 females (11 sterilised) and 40 males ( 7 castrated), aged 8-192 months, weighing 3.5-14 kg.

A complete physical examination was performed with special attention to a thorough thoracic auscultation lasting 2-3 min to rule out any subtle heart murmurs. Auscultation was performed in a fairly large examination room of about $20 \mathrm{~m}^{2}$ while ensuring a maximally quiet environment to guarantee minimal sound artifacts. A paediatric stethoscope (3M Littmann Classic II Paediatric, 3M Health Care, USA) with a diaphragm diameter of $3.32 \mathrm{~cm}$ and a bell diameter of $2.54 \mathrm{~cm}$ was used. Next a transthoracic echocardiographic examination was carried out according to the standard protocol (21) without the use of chemical restraint, making use of ultrasound machines ((SC300 PANDION before September 2005 (Esaote, Italy) and Hitachi Aloka 4000 (Hitachi Aloka Medical, Japan), after this date)) equipped with cardiology programmes and 2.5-7 megahertz sector transducers. In some cases the examination was performed on dogs in the standing position $(9,11)$.

Although all standard measurements were recorded during the echocardiographic examination, for the purpose of this study only the presence or absence of mitral valve lesions and regurgitation was analysed. The mitral valve leaflets were assessed in twodimensional right parasternal long axis 4-chamber and left caudal 4-chamber views. Mitral regurgitation was assessed from the left caudal 4-chamber view using colour flow mapping of the mitral valve area and when possible using spectral Doppler velocity measurements. Mitral regurgitation assessment is often subjective and the best efforts were made to minimise any artifacts. Data for further assessment of possible regurgitant jet size, mitral valve prolapse (MVP), and leaflet thickening were available for 77 dogs; however, 37 dogs had normal echocardiographic findings, therefore, a semiquantitative estimate as previously described (12, $16,18)$ was performed for the 40 dogs with changes. For regurgitant jet size estimation the portion of the left atrial size that was occupied by the regurgitant jet was measured. For MVP the position of the mitral leaflets with respect to a line drawn between the two hinge points of the mitral annulus was considered, and for leaflet thickening the anterior leaflet was measured for maximal thickness in early systole (16). A ratio of jet area to left atrial area $<20 \%$ was considered mild regurgitation, $20 \%-40 \%$ - moderate regurgitation and $>40 \%$ - severe regurgitation (12). Mitral valve prolapse did not occur if the mitral leaflets did not touch the mitral annulus plane. When leaflets touched or slightly passed this annular plane, the prolapse was considered mild. Prolapse was considered as moderate when the leaflets were displaced towards the left atrium and passed the mitral annulus plane, but did not pass a line drawn from the lowest point of the atrial septal membrane at the junction of the interventricular septum to the lower portion of the left atrial free wall at the junction of the left ventricular free wall. Leaflets passing this last line were considered severe prolapse (18). Leaflet thickness was measured in millimetres and under $2 \mathrm{~mm}$ was accepted as normal (16). All examinations were carried out by one of two veterinarians at the Cardiology Service of the Small Animal Clinic at the Faculty of Veterinary Medicine, Warsaw University of Life Sciences, Warsaw.

Statistical analysis was performed with the SPSS 21.0 (IBM, USA) programme. The frequency of mitral valve regurgitation (MVR) depending on age and gender was analysed using the Chi-squared test and Fisher's exact test. Descriptive statistical analysis was also performed (Microsoft Excel).

\section{Results}

Echocardiographic evidence of mitral regurgitation was seen in 68 Dachshunds (63.6\%) without heart murmurs. The percentage of dogs with mitral valve regurgitation increased significantly in Dachshunds over 5 years of age $(\mathrm{P}<0.0)$ with the highest percentage seen in Dachshunds older than 10 years $(57.4 \%)$ (Table 2). A larger percentage of males had mitral regurgitation (31 out of 41 examined males, $75.6 \%$ ) compared to the percentage of affected females (37 out of 66 examined females, 56.1\%) (Table 2). Descriptive analysis of the 40 affected dogs (Table 1) showed the mitral regurgitant jet size to be small in most dogs (average 16.2\%, range 0\%-45\%), mitral valve prolapse to be mild in most dogs $(72.5 \%)$, and thickening of the mitral leaflet to be minimal (average $3.3 \mathrm{~mm}$, range $0-6.2 \mathrm{~mm}$ ). Further statistical analysis of these parameters was not performed due to the small number of data points.

There were no statistically significant differences seen when comparing the influence of gender on different age groups of dogs (data not shown). However, analysis of the different age subgroups within the male and female groups showed statistically significant differences (Table 2). Female dogs became increasingly affected with mitral valve regurgitation with older age (from $5.4 \%$ in females below 5 years of age to $62.2 \%$ in females above 10 years of age). An increasing tendency to mitral valve regurgitation was also seen in males; however, this was not statistically significant, with MVR occurring in each age group. 
Table 1. Regurgitant jet size, mitral valve prolapse (MVP), and leaflet thickening in 40 dogs

\begin{tabular}{llllll}
\hline \multicolumn{2}{l}{ Mitral regurgitant jet size } & \multicolumn{2}{l}{ Leaflet thickening } & \multicolumn{2}{l}{ Mitral valve prolapse } \\
\hline & $\mathrm{n}=40$ & & $\mathrm{n}=40$ & & \\
\hline average & $16.2 \%$ & average & $3.3 \mathrm{~mm}$ & MVP 0 & 6 dogs \\
\hline median & $14.0 \%$ & median & $3.3 \mathrm{~mm}$ & MVP 1 & 29 dogs \\
\hline range & $0 \%-45 \%$ & range & $0-6.2 \mathrm{~mm}$ & MVP 2 & 3 dogs \\
\hline & & & MVP 3 & 2 dogs \\
\hline
\end{tabular}

MVP 0 - no prolapse; MVP 1 - mild prolapse; MVP 2 - moderate prolapse; MVP 3 - severe prolapse; $\mathrm{n}$ - number of animals

Table 2. The number of female and male Dachshunds with and without regurgitation in different age groups

\begin{tabular}{|c|c|c|c|c|}
\hline Gender & Age & $\begin{array}{l}\text { No mitral regurgitation } \\
\mathrm{n}(\%)\end{array}$ & $\begin{array}{ll}\text { Mitral regurgitation } \\
\mathrm{n}(\%)\end{array}$ & $\begin{array}{l}\text { Sum of dogs } \\
n(\%)\end{array}$ \\
\hline \multirow{4}{*}{$\begin{array}{l}\text { All dogs } \\
P=0.001\end{array}$} & $<5$ years & $14(35.9 \%)^{\mathrm{a}, \mathrm{b}}$ & $7(10.3 \%)^{a, b}$ & $21(19.6 \%)$ \\
\hline & $>5<10$ & $15(38.5 \%)^{\mathrm{a}, \mathrm{c}}$ & $22(32.4 \%)^{\mathrm{a}, \mathrm{c}}$ & $37(34.6 \%)$ \\
\hline & $>10$ & $10(25.6 \%)^{\mathrm{b}, \mathrm{c}}$ & $39(57.4 \%)^{\mathrm{b}, \mathrm{c}}$ & $49(45.8 \%)$ \\
\hline & Sum & $39(36.4 \%)$ & $68(63.6 \%)$ & $107(100.0 \%)$ \\
\hline \multirow{4}{*}{$\begin{array}{l}\text { Females } \\
P=0.003\end{array}$} & $<5$ years & $10(34.5 \%)$ & $2(5.4 \%)$ & $12(18.2 \%)$ \\
\hline & $>5<10$ & $11(37.9 \%)$ & $12(32.4 \%)$ & $23(34.8 \%)$ \\
\hline & $>10$ & $8(27.6 \%)$ & $23(62.2 \%)$ & $31(47.0 \%)$ \\
\hline & Sum & $29(43.9 \%)$ & $37(56.1 \%)$ & $66(100.0 \%)$ \\
\hline \multirow{4}{*}{$\begin{array}{l}\text { Males } \\
P=0.169\end{array}$} & $<5$ years & $4(40.0 \%)$ & $5(16.1 \%)$ & $9(22.0 \%)$ \\
\hline & $>5<10$ & $4(40.0 \%)$ & $10(32.3 \%)$ & $14(34.1 \%)$ \\
\hline & $>10$ & $2(20.0 \%)$ & $16(51.6 \%)$ & $18(43.9 \%)$ \\
\hline & Sum & $10(24.4 \%)$ & $31(75.6 \%)$ & $41(100.0 \%)$ \\
\hline
\end{tabular}

${ }^{\mathrm{a}} \mathrm{P}=0.1-$ a significant difference in the incidence of regurgitation between dogs in the $<5$ year and $>5<10$ year age groups

${ }^{\mathrm{b}} \mathrm{P}<0.0$ - a significant difference in the incidence of regurgitation between dogs in the $<5$ year and $>10$ year age groups

${ }^{\mathrm{c}} \mathrm{P}=0.042-\mathrm{a}$ significant difference in the incidence of regurgitation between dogs in the $>5<10$ and $>10$ year age groups

$\mathrm{n}$ - number of dogs

\section{Discussion}

The increased number of affected older Dachshunds is similar to what others have published (5, $6,20)$. Also consistent with other publications, this study shows that males continued to have a significantly higher prevalence of mitral regurgitation than females, especially at a younger age $(6,8,16,22)$. Studies have shown that there is an increase in heart murmur intensity with increased chronic mitral valve disease severity $(3,4,13)$. However, information is lacking regarding the scale of mitral regurgitation in dogs without auscultatory changes.

The present study shows that over $40 \%$ of dogs with MVR are under 10 years of age. Although most dogs with CMVD do not progress to more advanced stages of congestive heart failure, it is beneficial to know that the disease is present, especially for scientific studies. This is particularly important when taking into consideration the shift in the trend of scientific research from clinical to biomedical research.

Available published data show the onset of clinical CMVD to occur in older dogs $(19,20)$. Serfass et al. (20) have proposed a calculated theoretical age of 9.5 years (114 months) as the age when $20 \%$ of Dachshunds will have a murmur related to mitral valve disease, which may serve as an orientation point for clinically relevant mitral valve disease, but not for subclinical disease. This study, in conjunction with Olsen et al. (16) showing early mitral valve prolapse in Dachshunds, shows that the disease originates much earlier. Echocardiographic studies should therefore be performed in study models even if heart murmurs are absent.

The Cavalier King Charles spaniel has been categorised as an exception in that chronic mitral valve disease in this breed may appear at a very early age (5, $8,15)$. These dogs often present with early onset heart murmurs as a prequel to symptomatic valvular heart disease. Breeding programmes based on thoracic auscultatory findings have been instituted in this breed in hopes of delaying the onset of CMVD and/or decreasing the prevalence of the disease. There is conflicting information regarding the benefits of these breeding programmes. One study showed no effect of selecting dogs without heart murmurs for breeding (14), while another study showed that in fact the number of affected dogs dropped when a selection programme was in effect (2). The question remains whether we could further decrease the prevalence of CMVD if echocardiography, not auscultation, was used as a screening tool and a recent study shows that this is in fact possible (2).

This study shows that the prevalence of mitral valve regurgitation is underestimated when looking 
solely at dogs with heart murmurs. Echocardiographic studies in dog breeds predisposed to chronic mitral valve disease without heart murmurs are warranted first of all as a preventative diagnostic method for heart disease if we are to eliminate suspicion of subclinical chronic mitral valve disease, and secondly to enable a better global picture of the prevalence of the disease since studies of dogs with heart murmurs underestimate the number of affected animals.

The drawbacks of this study include its retrospective character, a lack of longitudinal followup, and the small number of dogs in different age groups which makes it impossible to more precisely analyse the effect of age on the presence of mitral regurgitation. Also, the study did not allow for distinction between acquired mitral valve disease and congenital valve defects such as mitral valve dysplasia, but rather relied on previously published data on the prevalence of CMVD in Dachshunds.

Conflict of Interests Statement: The authors declare that there is no conflict of interests regarding the publication of this article.

Financial Disclosure Statement: This study was supported by grant no. N N308 607538 received from the Polish Ministry of Science and Higher Education.

Animal Rights Statement: The study complies with national and institutional guidelines regarding the use of animals in clinical research according to the Polish legal act of January $21^{\text {st }}$, 2005. All owners gave consent for inclusion of their pet's data in the study.

Acknowledgements: The authors wish to thank Prof. Włodzimierz Kluciński for his support and help in the final preparation of this manuscript.

\section{References}

1. Abbott J.A.: Acquired Valvular Disease. In: Manual of Canine and Feline Cardiology. edited by Tilley, Smith, Oyama, Sleeper, Saunders Elsevier, St. Louis, 2008, pp. 110-138.

2. Birkegård A.C., Reinmann M.J., Martinussen T., Häggström J., Pedersen H.D., Olsen L.H.: Breeding restrictions decrease the prevalence of myxomatous mitral valve disease in Cavalier King Charles spaniels over an 8- to 10 -year period. J Vet Intern Med 2016, 30, 63-68.

3. Borgarelli M., Crosara S., Lamb K., Savarino P., La Rosa G., Tarducci A., Häaggstrom J.: Survival characteristics and prognostic variables of dogs with preclinical chronic degenerative mitral valve disease attributable to myxomatous degeneration. J Vet Intern Med 2011, 26, 69-75.

4. Borgarelli M., Savarino P., Crosara S., Santilli R.A., Chiavegato D., Poggi M., Bellino C., La Rosa G., Zanatta R., Häggström J., Tarducci A.: Survival characteristics and prognostic variables of dogs with mitral regurgitation attributable to myxomatous valve disease. J Vet Intern Med 2008, 22, 120-128.

5. Chetboul V., Tissier R., Villaret F., Nicolle A., Dean E., Benalloul T., Pouchelon J.L.: Epidemiological, clinical, echo-
Doppler characteristics of mitral valve endocardiosis in Cavalier King Charles in France: a retrospective study of 451 cases (1995 to 2003). Can Vet J 2004, 45, 1012-1015.

6. Egenvall A., Bonnett B.N., Häggström J.: Heart disease as a cause of death in insured Swedish dogs younger than 10 years of age. J Vet Intern Med 2006, 20, 894-903.

7. Garncarz M., Parzeniecka-Jaworska M., Jank M., Łój M.: Epidemiology of heart disease in a group of 207 Dachshund dogs in Poland - retrospective study. Acta Vet Scand 2013, doi:10.1186/1751-0147-55-52.x.

8. Häggström J., Hansson K., Kvart C., Swenson L.: Chronic valvular disease in the Cavalier King Charles spaniel in Sweden. Vet Rec 1992, 131, 549-553.

9. Häggström J., Kvart C., Pedersen H.D.: Acquired valvular disease. In: Textbook of Veterinary Internal Medicine. Edited by Ettinger S., Feldman E.C., St Louis, Elsevier Saunders; 2005. pp. 1022-1039.

10. Hulanicka M., Garncarz M., Parzeniecka-Jaworska M., Jank M. Plasma miRNAs as potential biomarkers of chronic degenerative valvular disease in Dachshunds. BMC Vet Res 2014, 10, 205-212.

11. Kittleson M.D.: Myxomatous atrioventricular valvular degeneration. In: Small Animal Cardiovascular Medicine. edited by Kittleson, Kienle, Mosby, Inc., Missouri, 1998, pp. 297-317.

12. Kossaify A., Akiki V.: Echocardiographic assessment of mitral valve regurgitation, pattern and prevalence, expanding clinical awareness through an institutional survey with the perspective of a quality improvement project. Clin Med Insights Cardiol 2014, 8, 71-77.

13. Lewis T., Swift S., Woolliams J.A., Blott S.: Heritability of premature mitral valve disease in Cavalier King Charles spaniels. Vet J 2011, 188, 73-76.

14. Lundin T., Kvart C.: Evaluation of the Swedish breeding program for cavalier King Charles spaniels. Acta Vet Scand 2010, 52, 54-59.

15. Malik R., Hunt G.B., Allan G.S.: Prevalence of mitral valve insufficiency in Cavalier King Charles spaniels. Vet Rec 1992, 130, 302-303.

16. Olsen L.H., Fredholm M., Pedersen H.D.: Epidemiology and inheritance of mitral valve prolapse in Dachshunds. J Vet Intern Med 1999, 13, 448-456.

17. Pasławska U., Noszczyk-Nowak A., Okręglicka E., Nicpoń J.: Analysis of arrhythmia of the heart in dogs examined between 1996-2001 in the Clinic of Horse, Dog and Cat Diseases at the Faculty of Veterinary Medicine of Wroclaw Agricultural University. Med Weter 2004, 60, 1191-1195.

18. Pedersen H., Häggstrom J.: Mitral valve prolapse in the dogs. Cardiovasc Res 2000, 47, 234-243.

19. Pedersen H.D., Kristensen B.O., Norby B., Lorentzen K.A.: Echocardiographic study of mitral valve prolapse in Dachshunds. J Vet Med A 1996, 43, 103-110.

20. Serfass P., Chetboul V., Sampedrano C.C., Nicolle A., Benalloul T., Laforge H., Gau C., Hebert C., Pouchelon J.L., Tissier R.: Retrospective study of 942 small sized dogs: prevalence of left apical systolic heart murmur and left-sided heart failure, critical effects of breed and sex. J Vet Cardiol 2006, $8,11-18$.

21. Thomas W.P., Gaber C.E., Jacobs G.J., Kaplan P.M., Lombard C.W., Moise N.S., Moses B.L.: Recommendations for standards in transthoracic two dimensional echocardiography in the dog and cat. J Vet Intern Med 1993, 7, 247-252.

22. Thrusfield M.V., Aitken C.G.G., Darke P.G.G.: Observations on breed and sex in relation to canine heart valve incompetence. J Small Anim Pract 1985, 26, 709-717.

23. Trafny D.J., Freeman L.M., Bulmer B.J., MacGregor J.M., Ruch J.E., Meurs K.M., Oyama M.A.: Auscultatory, echocardiographic, biochemical, nutritional, and environmental characteristics of mitral valve disease in Norfolk terriers. J Vet Cardiol 2012, 14, 261-267. 